\title{
Kybernetika
}

\section{Vojtech Veselý}

Design of robust output affine quadratic controller

Kybernetika, Vol. 40 (2004), No. 2, [221]--232

Persistent URL: http://dml.cz/dmlcz/135590

\section{Terms of use:}

(C) Institute of Information Theory and Automation AS CR, 2004

Institute of Mathematics of the Academy of Sciences of the Czech Republic provides access to digitized documents strictly for personal use. Each copy of any part of this document must contain these Terms of use.

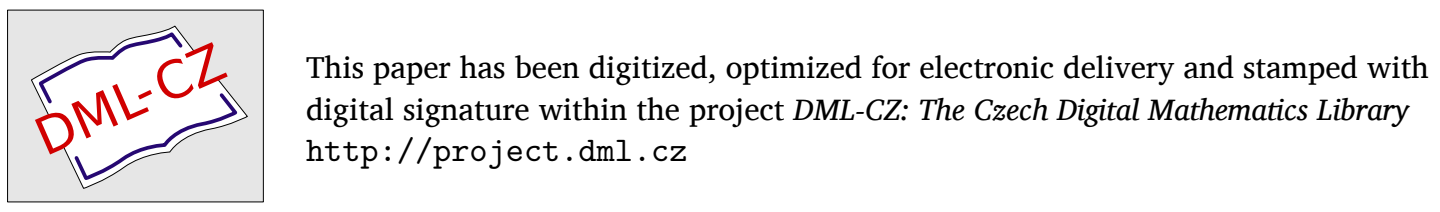




\title{
DESIGN OF ROBUST OUTPUT AFFINE QUADRATIC CONTROLLER ${ }^{1}$
}

\author{
VOJTECH VESELÝ
}

The paper addresses the problem robust output feedback controller design with guaranteed cost and affine quadratic stability for linear continuous time affine systems. The proposed design method leads to a non-iterative LMI based algorithm. A numerical example is given to illustrate the design procedure.

Keywords: robust control, parameter dependent Lyapunov function, affine quadratic stability, LMI approach

AMS Subject Classification: 93D15

\section{INTRODUCTION}

Robustness has been recognized as a key issue in the analysis and design of control systems for the last two decades. During the last decades numerous papers dealing with the design of static robust output feedback control schemes to stabilize uncertain systems have been published, Benton and Smith [1], Crusius and Trofino [3], Ghaoui and Balakrishnan [4], Geromel, De Souza, and Skelton [7], Gyurkovics and Takacs [9], Hejdiš and et al [10], Henrion and et al [11], Kose and Jabbari [12], Kozáková [13], Li Yu and Jian Chu [15], Mehdi, Al Hamid and Perrin [16], Pakshin [18], Pogyeon and et al [19], Tuan and et al [21], Xu and Darouch [24], Yong Yan Cao and You Xian Sun [25]. Various approaches have been used to study the two aspects of the robust stabilization problem, namely conditions under which the linear system described in state space can be stabilized via output feedback and the respective procedure to obtain a stabilizing or robustly stabilizing control law.

The necessary and sufficient conditions to stabilize the linear continuous time invariant system via static output feedback can be found in Kučera and De Souza [14] and in Veselý [22]. In the above and other papers, the authors basically conclude that despite the availability of many approaches and numerical algorithms the static output feedback problem is still open.

Recently, it has been shown that an extremely wide array of robust controller design problems can be reduced to the problem of finding a feasible point under a

\footnotetext{
${ }^{1}$ The first version of this paper has been presented at IFAC 15th World Congress, Barcelona 2002.
} 
Biaffine Matrix Inequality (BMI) constraint. The BMI has been introduced in Goh, Safonov and Papavassilopoulos [8]. In this paper, the BMI problem of robust controller design with output feedback is reduced to a LMI problem, Boyd, El Ghaoui, Feron and Balakrishnan [2]. The theory of Linear Matrix Inequalities has been used to design robust output feedback controllers in Benton and Smith [1], Crusius and Trofino [3], El Ghaoui and Balakrishnan [4], Henrion and et al [11], Li Yu and Jian Chu [15], Tuan, Apkarian, Hosoe and Tuy [21], Veselý [22]. Most of the above works present iterative algorithms in which a set of LMI problems are repeated until certain convergence criteria are met. The V-K iteration algorithm, proposed in $\mathrm{El}$ Ghaoui and Balakrishnan [4], is based on an alternative solution of two convex LMI optimization problems obtained by fixing the Lyapunov matrix or the gain controller matrix. This algorithm is guaranteed to converge, but not necessarily, to the global optimum of the problem depending on the starting conditions.

In this paper, new necessary and sufficient conditions to stabilize continuous time systems via static output feedback have been used to design a robust affine controller. For guaranteed cost and affine system this leads to a non iterative LMI based algorithm. The design procedure guarantees with sufficient conditions the robust affine quadratic stability for closed loop systems.

The paper is organized as follows. In Section 2 the problem formulation and some preliminary results are brought. The main results are given in Section 3 . In Section 4 the obtained theoretical results are applied.

We have used the standard notation. A real symmetric positive (negative) definite matrix is denoted by $P>0(P<0)$. Much of the notation and terminology follows the references of Kučera and De Souza [14], and Gahinet, Apkarian and Chilali [5].

\section{PRELIMINARIES AND PROBLEM FORMULATION}

This paper is concerned with the class of uncertain linear systems that can be described as

$$
\begin{aligned}
\dot{x}(t) & =\left(A_{c 0}+A_{c 1} \theta_{1}+\ldots+A_{c k} \theta_{k}\right) x(t) \\
& =A_{c}(\theta) x(t)
\end{aligned}
$$

where $\theta=\left[\theta_{1}, \ldots, \theta_{k}\right] \in \mathbb{R}^{k}$ is a vector of uncertain and possibly time varying real parameters.

The system represented by(1) is a polytope of linear affine systems which can be described by a list of its vertices

$$
\dot{x}(t)=D_{c i} x(t), \quad i=1,2, \ldots, N
$$

where $N=2^{k}$.

The system represented by (2) is quadratically stable if and only if there is a Lyapunov matrix $P>0$ such that

$$
D_{c i}^{T} P+P D_{c i}<0, \quad i=1,2, \ldots, N .
$$

A weakness of quadratic stability is that it guards against arbitrary fast parameter variations. As a result, this test tends to be conservative for constant or slow-varying 
parameters $\theta$. To reduce conservatism when (1) is affine in $\theta$ and the parameters of system are time invariant, in Gahinet, Apkarian and Chilali [5], the parameterdependent Lyapunov functions $P(\theta)$ has been used in the form

$$
P(\theta)=P_{0}+P_{1} \theta_{1}+\ldots+P_{k} \theta_{k}
$$

Other types of parameter-dependent Lyapunov functions have been proposed in De Oliviera, Bernussou and Geromel [17] for the stability analysis of linear discrete time systems and for the analysis and the design of continuous time systems with affine type uncertainties in Henrion, Alzelier and Peaucelle [11] and in Takahashi, Ramos, and Peres [20].

We shall consider the following linear time invariant continuous time uncertain systems

$$
\begin{aligned}
& \dot{x}(t)=A(\theta) x(t)+B(\theta) u(t) \\
& y(t)=C(\theta) x(t), \quad x(0)=x_{0}
\end{aligned}
$$

where $x(t) \in \mathbb{R}^{n}$ is the plant state; $u(t) \in \mathbb{R}^{m}$ is the control input; $y(t) \in \mathbb{R}^{\ell}$ is the output vector of system; $A(\theta), B(\theta), C(\theta)$ are matrices of appropriate dimensions depending affinely on $\theta$

$$
\begin{aligned}
& A(\theta)=A_{0}+A_{1} \theta_{1}+\ldots+A_{k} \theta_{k} \\
& B(\theta)=B_{0}+B_{1} \theta_{1}+\ldots+B_{k} \theta_{k} \\
& C(\theta)=C_{0}+C_{1} \theta_{1}+\ldots+C_{k} \theta_{k} .
\end{aligned}
$$

Note that, in order to keep the polytope affine property, the matrix $B(\theta)$ or $C(\theta)$ must be precisely known. The following definition and theorem by Gahinet, Apkarian and Chilali [5] will be heavily exploited in the next development.

Definition 1. The linear system

$$
\dot{x}(t)=A_{c}(\theta) x(t), \quad x(0)=x_{0}
$$

is affine quadratically stable if there exist $k+1$ symmetric matrices $P_{0}, P_{1}, \ldots, P_{k}$ such that

$$
P(\theta)=P_{0}+P_{1} \theta_{1}+\ldots+P_{k} \theta_{k}>0
$$

and

$$
\frac{\mathrm{d} V(x, \theta)}{\mathrm{d} t}=x(t)^{T}\left(A_{c}^{T}(\theta) P(\theta)+P(\theta) A_{c}(\theta)+\frac{\mathrm{d} P(\theta)}{\mathrm{d} t}\right) x(t)<0
$$

for $\theta=\left[\theta_{1}, \ldots, \theta_{k}\right]$.

Note that quadratic stability corresponds to the case $P_{1}=\ldots=P_{k}=0$. Sufficient affine quadratic stability conditions are given by the next theorem. 
Theorem 1. (Gahinet, Apkarian and Chilali [5].) Consider the linear systems governed by (6), where $A_{c}(\theta)$ depends affinely on the uncertain parameter vector $\theta=\left[\theta_{1}, \ldots, \theta_{k}\right]$ and $\theta_{i}$ satisfies

$$
\theta_{i} \in\left\langle\underline{\theta}_{i}, \bar{\theta}_{i}\right\rangle, \quad \dot{\theta}_{i} \in\left\langle\underline{\nu}_{i}, \bar{\nu}_{i}\right\rangle, \quad \text { for } i=1,2, \ldots, k
$$

where $\underline{\theta}_{i}, \bar{\theta}_{i}, \underline{\nu}_{i}, \bar{\nu}_{i}$ are known lower and upper bounds. Let $\Gamma$ and $\Lambda$ denote the set of $N=2^{k}$ vertices of the parameters box (9) and of the rate of variation box (9), respectively

$$
\begin{array}{cccc}
\Gamma & =\left\{\left(\gamma_{1}, \ldots, \gamma_{k}\right): \gamma_{i}=\underline{\theta}_{i}\right. & \text { or } & \left.\gamma_{i}=\bar{\theta}_{i}\right\} \\
\Lambda & =\left\{\left(\lambda_{1}, \ldots, \lambda_{k}\right): \lambda_{i}=\underline{\nu}_{i}\right. & \text { or } & \left.\lambda_{i}=\bar{\nu}_{i}\right\}
\end{array}
$$

and let

$$
\theta_{m}=\left[\frac{\underline{\theta}_{1}+\bar{\theta}_{1}}{2}, \ldots, \frac{\underline{\theta}_{k}+\bar{\theta}_{k}}{2}\right]
$$

denote the average value of the uncertain parameters vector. This system is affine quadratically stable if $A_{c}\left(\theta_{m}\right)$ is stable and there exist $k+1$ symmetric matrices $P_{0}, P_{1}, \ldots, P_{k}$ such that $P(\theta)>0$ satisfies

$$
L(\gamma, \lambda)=A_{c}(\gamma)^{T} P(\gamma)+P(\gamma) A_{c}(\gamma)+P(\lambda)-P_{0}<0
$$

for all $(\gamma, \lambda) \in \Gamma \times \Lambda$ and

$$
A_{c i}^{T} P_{i}+P_{i} A_{c i} \geq 0
$$

for $i=1,2, \ldots, k$.

When (11) and (12) are met, a Lyapunov function for (6) and all trajectories $\theta(t)$ satisfying (9) is given by

$$
V(x, \theta)=x^{T}(t) P(\theta) x(t) .
$$

The following performance index is associated with the system (5)

$$
J=\int_{0}^{\infty}\left(x(t)^{T} Q x(t)+u(t)^{T} R u(t)\right) \mathrm{d} t
$$

where $Q=Q^{T} \geq 0, R=R^{T}>0$ are matrices of compatible dimensions.

The problem studied in this paper can be formulated as follows:

For a continuous time system described by (5) design a static output feedback controller with the gain matrix $F$ and control algorithm

$$
u(t)=F y(t)=F C(\theta) x(t)
$$

so that the closed loop system

$$
\dot{x}=(A(\theta)+B(\theta) F C(\theta)) x(t)=A_{c}(\theta) x(t)
$$

is affine quadratically stable with guaranteed cost. 
Definition 2. Consider the system (5). If there exists a control law $u^{*}$ and a positive scalar $J^{*}$ such that closed loop system (15) is stable and the closed loop value cost function (13) satisfies $J \leq J^{*}$, then $J^{*}$ is said to be the guaranteed cost and $u^{*}$ is said to be the guaranteed cost control law for system (5).

\section{THE MAIN RESULTS}

In this section we present a new procedure to design a static output feedback controller for affine continuous time linear systems (5) which ensure the guaranteed cost and affine quadratic stability of closed loop system. The following theorem is one of the main results.

Theorem 2. For system (5) and Lyapunov function $V(\theta)=x(t)^{T} P(\theta) x(t)>0$ the following statements are equivalent:

- There exist positive definite matrices $Q$ and $R$ that system (5) is static output feedback affine quadratic stabilizable (AQS) with guaranteed cost

$$
\int_{0}^{\infty} x(t)^{T}\left(Q+C(\theta)^{T} F^{T} R F C(\theta)\right) x(t) \mathrm{d} t \leq x_{0}^{T} P(\theta) x_{0} .
$$

- There exist $k+1$ symmetric matrices $P_{0}, P_{1}, \ldots, P_{k}$ that $P(\theta)>0$, positive definite matrices $Q$ and $R$, and matrix $F$ such that the following inequality holds

$$
\begin{gathered}
(A(\theta)+B(\theta) F C(\theta))^{T} P(\theta)+P(\theta)(A(\theta)+B(\theta) F C(\theta))+\dot{P}(\theta) \\
+Q+C(\theta)^{T} F^{T} R F C(\theta)<0 .
\end{gathered}
$$

- There exist $k+1$ symmetric matrices $P_{0}, P_{1}, \ldots, P_{k}$ that (7) holds, positive definite matrices $Q$ and $R$, and matrix $F$ such that the following inequality holds

$$
\begin{gathered}
A(\theta)^{T} P(\theta)+P(\theta) A(\theta)-P(\theta) B(\theta) R^{-1} \\
B(\theta)^{T} P(\theta)+\dot{P}(\theta)+Q+G(\theta)^{T} R^{-1} G(\theta)<0
\end{gathered}
$$

where

$$
G(\theta)=B(\theta)^{T} P(\theta)+R F C(\theta) .
$$

- There exist $k+1$ symmetric matrices $P_{0}, P_{1}, \ldots, P_{k}$ that (7) holds, positive definite matrices $Q$ and $R$, and matrix $F$ such that the following inequality holds

$$
\begin{gathered}
A(\theta)^{T} P(\theta)+P(\theta) A(\theta)-P(\theta) B(\theta) R^{-1} B(\theta)^{T} P(\theta)+P(\theta)+Q<0 \\
G(\theta) \phi(\theta)^{-1} G(\theta)^{T}-R<0
\end{gathered}
$$

where

$$
\begin{gathered}
\phi(\theta)=A(\theta)^{T} P(\theta)+P(\theta) A(\theta) \\
-P(\theta) B(\theta) R^{-1} B(\theta)^{T} P(\theta)+\dot{P}(\theta)+Q .
\end{gathered}
$$


The proof of this theorem goes the same way as in [22, 23].

Because of Theorem 1, inequalities (17), (18) and (19), (20) are negative in the box (10) if they take negative values at the vertices of (10); that is if they are negative for all $\gamma$ in the vertex set $\Gamma$ given by (10) and inequality (12) holds for all $i=1,2, \ldots, k$. In the vertex set (10) define the polytope system in the form

$$
\left\{\left(D_{1}, E_{1}, H_{1}\right), \ldots,\left\{D_{N}, E_{N}, H_{N}\right)\right\}
$$

where $N=2^{k}$ and

$$
\begin{aligned}
& D_{1}=A_{0}+A_{1} \underline{\theta}_{1}+\ldots+A_{k} \underline{\theta}_{k} \\
& E_{1}=B_{0}+B_{1} \underline{\theta}_{1}+\ldots+B_{k} \underline{\theta}_{k} \\
& H_{1}=C_{0}+C_{1} \underline{\theta}_{1}+\ldots+C_{k} \underline{\theta}_{k} .
\end{aligned}
$$

In (21) each vertex is calculated for the $k$ variables $\theta_{i}, i=1,2, \ldots, k$ alternatively taken at maximum and minimum values. For polytopic system inequality (19) could not be solved within LMI therefore let us introduce the inverse Lyapunov matrix $S(\theta)$ as in Gahinet, Nemirovski, Laub and Chilali [6].

$$
S(\theta)=P(\theta)^{-1}=S_{0}+S_{1} \theta_{1}+\ldots+S_{k} \theta_{k} .
$$

Note that equality (23) holds for all vertices $(\gamma, \lambda) \in \Gamma \times \Lambda$. For the new variable $S(\theta)$, equations (11), (12) and (19) read as follows

$$
\begin{gathered}
L(\gamma, \lambda)=S(\gamma) A(\gamma)^{T}+A(\gamma) S(\gamma)-\left(S(\lambda)-S_{0}\right)<0 \\
S_{i} A_{c i}^{T}+A_{c i} S_{i} \geq 0, \quad i=1,2, \ldots, k
\end{gathered}
$$

and

$$
\begin{gathered}
S(\gamma) A(\gamma)^{T}+A(\gamma) S(\gamma)-B(\gamma) R^{-1} B(\gamma)^{T} \\
-\left(S(\lambda)-S_{0}\right)+S(\gamma) Q S(\gamma)<0 .
\end{gathered}
$$

For reducing the conservatism of the AQS test [5] nonnegative matrices $M_{i} \geq 0$, $i=1,2, \ldots, k$ are added to (26) and (25) as follows

$$
\begin{gathered}
S(\gamma) A(\gamma)^{T}+A(\gamma) S(\gamma)-B(\gamma) R^{-1} B(\gamma)^{T} \\
-\left(S(\lambda)-S_{0}\right)+S(\gamma) Q S(\gamma)+\sum_{i=1}^{k} \theta_{i}^{2} M_{i}<0
\end{gathered}
$$

and

$$
S_{i} A_{c i}^{T}+A_{c i} S_{i}+M_{i} \geq 0, \quad i=1,2, \ldots, k .
$$

The resulting test is generally less conservative for (27) and (28). However, this improvement is at the expense of higher computational needs since the number of optimization variables is increased in the new LMI problem (27), (28). Combining the results of (19), (27), (28) and (20) the following algorithm for computation of a robust output feedback controller with guaranteed affine quadratic stability has been proposed. 


\section{Algorithm.}

Step 1. Find the solution of (27) at all vertices $(\gamma, \lambda) \in \Gamma \times \Lambda$ with respect to the variables $S_{0}, S_{1}, \ldots, S_{k}, M_{1}, M_{2}, \ldots, M_{k}$ from the following LMI inequalities

$$
\left[\begin{array}{ccc}
N_{i}(\gamma, \lambda) & S(\gamma) Q & V_{i}(\gamma) \\
Q S(\gamma) & -Q & 0 \\
V_{i}^{T}(\gamma) & 0 & -R^{-1}
\end{array}\right] \leq 0
$$

where

$$
\begin{gathered}
N_{i}(\gamma, \lambda)=S(\gamma) D_{i}^{T}+D_{i} S(\gamma)-E_{i} R^{-1} E_{i}^{T}-\left(S(\lambda)-S_{0}\right)+\sum_{j=1}^{k} \theta_{j}^{2} M_{j} \\
V_{i}(\gamma)=\left(E_{i}^{T}+R F_{v} H_{i} S(\gamma)\right)^{T} R^{-1}
\end{gathered}
$$

for $i=1,2, \ldots, N$

$$
\begin{gathered}
K_{j}=S_{j} A_{j}^{T}+A_{j} S_{j}+M_{j} \geq 0 \\
M_{j} \geq \rho_{1} I, S(\gamma) \geq \rho_{2} I, S_{j} \leq \rho_{3} I, j=1,2, \ldots, k
\end{gathered}
$$

where $I$ is identity matrix with corresponding dimensions and $\rho_{1}, \rho_{2}, \rho_{3}$ are some nonnegative constants. For the first iterative procedure $F_{v}=0$.

Step 2. Calculate the value of the inverse Lyapunov matrix $S_{n i}$ and $P_{n i}, i=$ $1,2, \ldots, N$ at all vertices of $\gamma \in \Gamma$.

Step 3. Compute the value of Riccati equation at all vertices of $(\gamma, \lambda) \in \Gamma \times \Lambda$.

$$
\phi_{i}(\lambda)=D_{i}^{T} P_{n i}+P_{n i} D_{i}-P_{n i} E_{i} R^{-1} E_{i}^{T} P_{n i}+\left(P(\lambda)-P_{0}\right)+Q .
$$

Step 4. Compute the gain matrix $F$ from the following LMI inequalities

$$
\begin{gathered}
{\left[\begin{array}{cc}
-R & E_{i}^{T} P_{n i}+R F H_{i} \\
\left(E_{i}^{T} P_{n i}+R F H_{i}\right)^{T} & \phi_{i}(\lambda)
\end{array}\right] \leq 0} \\
i=1,2, \ldots, N \text { and } \lambda \in \Lambda \\
K_{j}+S_{j} C_{j}^{T} F^{T} B_{j}^{T}+B_{j} F C_{j} S_{j} \geq 0
\end{gathered}
$$

for $j=1,2, \ldots, k$.

If the solutions are feasible for designer then stop, else $F_{v}=F$ and go to Step 1 . Usually, the repeated procedure generates less conservative results than first one. The convergence of the above special iterative procedure has not been proven yet, however if the argument of [4] that $\mathrm{V}-\mathrm{K}$ iterative procedure is guaranteed to converge is taken into account we can conclude that the proposed algorithm is guaranteed to converge too, but not necessarily to the global optimum of the problem depending on starting conditions. 
Note that for example $S(\gamma)$ in (23) reads for $i=1$ as follows

$$
S(\gamma)=S_{0}+S_{1} \underline{\theta}_{1}+S_{2} \underline{\theta}_{2}+\ldots
$$

If the LMI problems (29) - (33) are feasible, the resulting gain matrix $F$ guarantees the affine quadratic stability and simultaneously ensures the guaranteed cost (16) for the closed loop system (15).

\section{EXAMPLES}

In this example we consider the linear model of two cooperating DC motors. The problem is to design two PI controllers for a laboratory MIMO system which will guarantee affine quadratic stability of a closed loop uncertain system. The system model is given by (5) with a time invariant matrix affine type uncertain structure, where

$$
\begin{aligned}
& A_{0}= {\left[\begin{array}{cccccccccc}
0 & -0.2148 & 0 & 0 & 0 & 0 & 0 & 0 & 0 & 0 \\
1 & -1.014 & 0 & 0 & 0 & 0 & 0 & 0 & 0 & 0 \\
0 & 0 & 0 & -0.2605 & 0 & 0 & 0 & 0 & 0 & 0 \\
0 & 0 & 1 & -0.9107 & 0 & 0 & 0 & 0 & 0 & 0 \\
0 & 0 & 0 & 0 & 0 & -0.1639 & 0 & 0 & 0 & 0 \\
0 & 0 & 0 & 0 & 1 & -0.8137 & 0 & 0 & 0 & 0 \\
0 & 0 & 0 & 0 & 0 & 0 & 0 & -0.2279 & 0 & 0 \\
0 & 0 & 0 & 0 & 0 & 0 & 1 & -0.8251 & 0 & 0 \\
0 & 1 & 0 & 1 & 0 & 0 & 0 & 0 & 0 & 0 \\
0 & 0 & 0 & 0 & 0 & 1 & 0 & 1 & 0 & 0
\end{array}\right] } \\
& A_{1}=\left[\begin{array}{cccccccccc}
0 & -0.025 & 0 & 0 & 0 & 0 & 0 & 0 & 0 & 0 \\
0 & -0.1395 & 0 & 0 & 0 & 0 & 0 & 0 & 0 & 0 \\
0 & 0 & 0 & -0.0938 & 0 & 0 & 0 & 0 & 0 & 0 \\
0 & 0 & 0 & -0.2911 & 0 & 0 & 0 & 0 & 0 & 0 \\
0 & 0 & 0 & 0 & 0 & 0.0188 & 0 & 0 & 0 & 0 \\
0 & 0 & 0 & 0 & 0 & 0.0208 & 0 & 0 & 0 & 0 \\
0 & 0 & 0 & 0 & 0 & 0 & 0 & -0.0333 & 0 & 0 \\
0 & 0 & 0 & 0 & 0 & 0 & 0 & -0.1173 & 0 & 0 \\
0 & 0 & 0 & 0 & 0 & 0 & 0 & 0 & 0 & 0 \\
0 & 0 & 0 & 0 & 0 & 0 & 0 & 0 & 0 & 0
\end{array}\right] \\
& A_{2}=\left[\begin{array}{cccccccccc}
0 & 0.0125 & 0 & 0 & 0 & 0 & 0 & 0 & 0 & 0 \\
0 & 0.0594 & 0 & 0 & 0 & 0 & 0 & 0 & 0 & 0 \\
0 & 0 & 0 & 0.0116 & 0 & 0 & 0 & 0 & 0 & 0 \\
0 & 0 & 0 & 0.0308 & 0 & 0 & 0 & 0 & 0 & 0 \\
0 & 0 & 0 & 0 & 0 & -0.0188 & 0 & 0 & 0 & 0 \\
0 & 0 & 0 & 0 & 0 & -0.0156 & 0 & 0 & 0 & 0 \\
0 & 0 & 0 & 0 & 0 & 0 & 0 & 0.0208 & 0 & 0 \\
0 & 0 & 0 & 0 & 0 & 0 & 0 & -0.0333 & 0 & 0 \\
0 & 0 & 0 & 0 & 0 & 0 & 0 & 0 & 0 & 0 \\
0 & 0 & 0 & 0 & 0 & 0 & 0 & 0 & 0 & 0
\end{array}\right]
\end{aligned}
$$




$$
\begin{aligned}
& B_{0}=\left[\begin{array}{cc}
0.3148 & 0 \\
0.0478 & 0 \\
0 & -0.1028 \\
0 & -0.0091 \\
-0.0841 & 0 \\
-0.0287 & 0 \\
0 & 0.3676 \\
0 & 0.2448 \\
0 & 0 \\
0 & 0
\end{array}\right] \quad B_{1}=\left[\begin{array}{cc}
0.0625 & 0 \\
-0.0798 & 0 \\
0 & -0.0462 \\
0 & -0.0449 \\
0.0016 & 0 \\
0.0072 & 0 \\
0 & 0.077 \\
0 & -0.005 \\
0 & 0 \\
0 & 0
\end{array}\right] \\
& B_{2}=\left[\begin{array}{cc}
-0.0094 & 0 \\
0.0151 & 0 \\
0 & 0.0019 \\
0 & -0.003 \\
-0.0121 & 0 \\
-0.03 & 0 \\
0 & -0.064 \\
0 & 0.0189 \\
0 & 0 \\
0 & 0
\end{array}\right] \quad C^{T}=\left[\begin{array}{llll}
0 & 0 & 0 & 0 \\
1 & 0 & 0 & 0 \\
0 & 0 & 0 & 0 \\
1 & 0 & 0 & 0 \\
0 & 0 & 0 & 0 \\
0 & 1 & 0 & 0 \\
0 & 0 & 0 & 0 \\
0 & 1 & 0 & 0 \\
0 & 0 & 1 & 0 \\
0 & 0 & 0 & 1
\end{array}\right]
\end{aligned}
$$

The number of polytope systems are equal to 4 and the polytope vertices are computed for two variables $\theta_{1}, \theta_{2}$ alternatively taken at their maximum $\bar{\theta}_{i}$ and minimum $\underline{\theta}_{i}, i=1,2$. The decentralized control structure for the two PI controllers can be obtained by the choice of the static output feedback gain matrix $F$ structure. It is given as follows

$$
F=\left[\begin{array}{cccc}
f_{11} & 0 & f_{13} & 0 \\
0 & f_{22} & 0 & f_{24}
\end{array}\right]
$$

The results of calculation of a static output feedback gain matrix $F$ for quadratically and affine quadratically stable system for different $Q=q I, R=r I,\left|\theta_{1}\right|=\left|\theta_{2}\right|=1$ and $\rho_{i}, i=1,2,3$ are summarized in the following table.

$\begin{array}{cccccccc}N & q & r & \rho_{1} & \rho_{2} & \rho_{3} & \text { quad } & \text { aff.quad } \\ 1 & 1.5 & 1 & 1.5 & 1 & 0.166 & -0.1342 & -0.0954 \\ 2 & 5 & 1 & 1.5 & 1 & 0.166 & +0.307 & -0.1277^{*} \\ 3 & 10 & 1 & 1.5 & 1 & 0.166 & -0.081 & -0.1922^{* *} \\ 4 & 20 & 1 & 1.5 & 1 & 0.166 & +1.11 & -0.1148 \\ 5 & 10^{-4} & 1 & 1.5 & 1 & 0.166 & -0.0164 & +0.0011 \\ 6 & 10^{-4} & 1 & 10^{-4} & 1 & 0.166 & -0.0164 & -0.0136 \\ 7 & 0.1 & 1 & 1.5 & 1 & 0.166 & -0.1383 & -0.0386 \\ 8 & 0.1 & 1 & 10^{-4} & 1 & 0.166 & -0.1383 & -0.1133 \\ 9 & 0.1 & 1 & 0 & 1 & 0.166 & -0.1383 & -0.1449 \\ 10 & 0.1 & 1 & 0 & 1 & 0 & -0.1383 & -0.1448 \\ 11 & 10^{-6} & 1 & 0 & 1 & 0.166 & -0.0015 & -0.0015 \\ 12 & 10^{-6} & 0.1 & 0 & 1 & 0.166 & -0.0134 & -0.0178\end{array}$

where quad and aff.quad denote the max (real (eigenvalue)) of the closed loop system for quadratic or affine quadratic stability, respectively. The solutions are feasible for 11 and 12 cases. For other cases the closed loop system is quadratically or affine 
quadratically stable but the minimal value of cost is not guaranteed. The static output feedback gain matrix for cases ${ }^{*}$ and ${ }^{* *}$ are given as follow

$$
\begin{aligned}
F^{*} & =\left[\begin{array}{cccc}
-0.3582 & 0 & -0.376 & 0 \\
0 & -0.7927 & 0 & -0.7535
\end{array}\right] \\
F^{* *} & =\left[\begin{array}{cccc}
-1.0708 & 0 & -0.6317 & 0 \\
0 & -2.6952 & 0 & -1.671
\end{array}\right]
\end{aligned}
$$

The second example has been borrowed from [1] to demonstrate the design of affine quadratic controller. It is known that the presented system is static output feedback stabilizable. Let $(A, B, C)$ in (5) be defined as

$$
\begin{gathered}
A=\left[\begin{array}{cccc}
-0.036 & 0.0271 & 0.0188 & -0.4555 \\
0.0482 & -1.010 & 0.0024 & -4.0208 \\
0.1002 & q_{1}(t) & -0.707 & q_{2}(t) \\
0 & 0 & 1 & 0
\end{array}\right] \\
B=\left[\begin{array}{cc}
0.4422 & 0.1761 \\
q_{3}(t) & -7.59222 \\
-5.520 & 4.490 \\
0 & 0
\end{array}\right] \quad C=\left[\begin{array}{llll}
0 & 1 & 0 & 0
\end{array}\right]
\end{gathered}
$$

with parameters bounds $-0.6319 \leq q_{1}(t) \leq 1.3681,1.22 \leq q_{2}(t) \leq 1.420$, and $2.7446 \leq q_{3}(t) \leq 4.3446$. Find a stabilizing output feedback matrix $F$. The nominal model of $\left(A_{0}, B_{0}\right)$ is given by the above matrices when we substitute for the entries $A_{0}(3,2)=0.3681, A_{0}(3,4)=1.32$ and $B_{0}(2,1)=3.5446$. The structured model uncertainty $(5)\left(A_{1}, A_{2}, B_{1}\right)$ are matrices with the following entries $A_{1}(3,2)=1, A_{2}(3,4)=0.1$ and $B_{1}(2,1)=0.8$ with $\theta_{i} \in\langle-1,1\rangle, i=1,2$. Other entries of the above uncertain matrices are equal to zero. The number of polytope systems are equal to 4 .

The nominal model is unstable with eigenvalues:

$$
\text { eig }\{-2.0516,0.2529 \pm 0.3247 \mathrm{i},-0.2078\}
$$

For the first "iterative" procedure, $F_{v}=0$, the closed-loop eigenvalues of 4 polytope systems are as follows

$$
\text { eig } C L\{0.6885,0.5726,0.0713 \pm 1.1077 \mathrm{i}, \ldots,-4.0422\} \text {. }
$$

Closed-loop system is not stable. After using the iterative procedure the closed-loop eigenvalues of 4 polytope systems are as follows

eig $C L\{-0.04504 \pm 0.3555 \mathrm{i},-0.1472 \pm 0.4304 \mathrm{i},-0.1896 \pm 0.4636 \mathrm{i}, \ldots,-688.527\}$.

The eigenvalues of closed-loop system for the case $\left\|\theta_{1}\right\|=\left\|\theta_{2}\right\|=0$ are equal as follows

$$
e i g C L\{-0.1668 \pm 0.4432 \mathrm{i},-0.403,-660.91\}
$$


and output feedback gain matrix

$$
F=\left[\begin{array}{c}
34.51 \\
103.03
\end{array}\right]
$$

Hence, the affine quadratic stability conditions are met the closed-loop system is affine quadratically stable. Because, the available solution in the above design LMI procedure is only may be feasible but are not strictly (best value of $t=5.17310^{-4}$ ) the cost minimal value (14) is not guaranteed. For the above example we obtain the value of cost as follows

$$
J=\int_{0}^{\infty}\left(x(t)^{T}\left(Q+C^{T} F^{T} R F C\right) x(t)\right) \mathrm{d} t \leq 33.82663\left\|x_{0}\right\|^{2} .
$$

\section{CONCLUSIONS}

In this paper, we have proposed a new procedure for robust output feedback controller design for linear systems with affine and possible time varying parameter uncertainty. The feasible solution of the output feedback controller with sufficient conditions guarantee the affine quadratic stability and guaranteed cost. The design procedure is based on new necessary and sufficient conditions for output feedback stabilizability of linear systems and a non-iterative LMI based algorithm. A valuable feature of the robust controller design procedure is that quantitative information about the rate of parameter variation is readily incorporated to reduce conservatism in the time varying case.

\section{ACKNOWLEDGEMENT}

The work has been supported by grant No. 1/0158/03 of the Scientific Grant Agency of the Ministry of Education of Slovak Republic and the Slovak Academy of Sciences.

The author is grateful to the referee for the careful reading of the manuscript and many helpful suggestions which improved the paper.

(Received October 3, 2002.)

\section{REFERENCES}

[1] R. E. Benton, Jr. and D. Smith: A non iterative LMI based algorithm for robust static output feedback stabilization. Internat. J. Control 72 (1999), 1322-1330.

[2] S. Boyd, L. El. Ghaoui, E. Feron, and V. Balakrishnan: Linear Matrix Inequalities in System and Control Theory. SIAM 115 (1994), Philadelphia.

[3] C. A.R. Crusius and A. Trofino: Sufficient LMI conditions for output feedback control problems. IEEE Trans. Automat. Control 44 (1999), 5, 1053-1057.

[4] L. El Ghaoui and V. Balakrishnan: Synthesis of fixed structure controllers via numerical optimization. In: Proc. 33rd Conference on Decision and Control, Lake Buena Vista, FL 1994, pp. 2678-2683.

[5] P. Gahinet, P. Apkarian, and M. Chilali: Affine parameter dependent Lyapunov functions and real parametric uncertainty. IEEE Trans. Automat. Control 41 (1996), 436442 . 
[6] P. Gahinet, A. Nemirovski, A. J. Laub, and M. Chilali: LMI Control Toolbox User's Guide. The Mathworks Inc., Natick MA 1995.

[7] J. C. Geromel, C.E. De Souza, and R. E. Skelton: Static output feedback controllers: stability and convexity. IEEE Trans. Automat. Control 43 (1998), 120-125.

[8] K. C. Goh, M. G. Safonov, and G.P. Papavassilopoulos: Global optimization for the biaffine matrix inequality problem. J. Global Optim. 7 (1995), 365-380.

[9] E. Gyurkovics and T. Takacs: Stabilisation of discrete-time interconnected systems under control constraints. Proc. IEE Control Theory and Applications 147 (2000), $137-144$

[10] J. Hejdiš, Š. Kozák, and L. Juráčková: Self-tuning controllers based on orthonormal functions. Kybernetika 36 (2000), 477-491.

[11] D. Henrion, D. Alzelier, and D. Peaucelle: Positive polynomial matrices and improved robustness conditions. In: Proc. 15th Triennial World Congres, Barcelona 2002, CD.

[12] I. E. Kose and F. Jabbari: Robust control of linear systems with real parametric uncertainty. Automatica 35 (1999), 679-687.

[13] A.Kozáková: Robust Decentralized control of complex systems in frequency domain. In: Preprints of 2nd IFAC Workshop on NTDCS, Elsevier Kidlington UK, 1999.

[14] V. Kučera and C. E. De Souza: A necessary and sufficient conditions for output feedback stabilizability. Automatica 31 (1995), 1357-1359.

[15] Li Yu and Jian Chu: An LMI approach to guaranteed cost of linear uncertain time delay systems. Automatica 35 (1999), 1155-1159.

[16] D. Mehdi, M. Al Hamid, and F. Perrin: Robustness and optimality of linear quadratic controller for uncertain systems. Automatica 32 (1996), 1081-1083.

[17] M. C. De Oliveira, J. Bernussou, and J. C. Geromel: A new discrete-time robust stability condition. Systems Control Lett. 37 (1999), 261-265.

[18] P. V. Pakshin: Robust decentralized control of systems of random structure. J. Computer and Systems Sciences 42 (2003), 200204.

[19] P. Park, Y.S. Moon, and W. H. Kwon: A stabilizing output feedback linear quadratic control for pure input delayed systems. Internat. J. Control 72 (1999), 385-391.

[20] R. H. C. Takahashi, D. C. W. Ramos, and P. L. D. Peres: Robust control synthesis via a genetic algorithm and LMIS. In: Preprints 15th Triennial World Congress, Barcelona 2002, CD.

[21] H. D. Tuan, P. Apkarian, S. Hosoe, and H. Tuy: D.C. optimization approach to robust control: feasibility problems. Internat. J. Control 73 (2000), 89-104.

[22] V. Veselý: Static output feedback controller design. Kybernetika 37 (2001), 205-221.

[23] V. Veselý: Robust output feedback controller design for linear parametric uncertain systems. J. Electrical Engineering 53 (2002), 117-125.

[24] S. J. Xu, and M. Darouch: On the robustness of linear systems with nonlinear uncertain parameters. Automatica 34 (1998), 1005-1008.

[25] Yong Yan Cao and You Xian Sun: Static output feedback simultaneous stabilization: LMI approach. Internat. J. Control 70 (1998), 803-814.

Vojtech Veselý, Department of Automatic Control Systems, Faculty of Electrical Engineering and Information Technology, Slovak University of Technology, Ilkovičova 3, 81219 Bratislava. Slovak Republic.

e-mail: vesely@kasr.elf.stuba.sk 